\title{
Utilidad de la integración y convergencia de los métodos cualitativos y cuantitativos en las investigaciones en salud*
}

\section{Usefulness of integration and convergence of qualitative and quantitative methods in health research}

\author{
Rosario García González \\ Investigadora Titular y de Mérito. Instituto Nacional de Endocrinología. La Habana, \\ Cuba.
}

\begin{abstract}
RESUMEN
El propósito del presente trabajo es comentar la posibilidad de la integración y convergencia de los métodos cuantitativo y cualitativo en las investigaciones en salud. Pretende, además, caracterizar ambos métodos y ejemplificarlo con investigaciones concretas en el campo de la salud. La información ofrecida se basa en la revisión bibliográfica, la exposición teórica y la presentación de resultados obtenidos en investigaciones sociales y educacionales en el campo de la diabetes. Los dos métodos tienen diferentes objetivos de investigación y forma de abordar el fenómeno a estudiar, sus ventajas y limitaciones. El método cuantitativo es útil cuando se quiere medir la magnitud de un fenómeno, el método cualitativo lo es cuando se quiere interpretar cómo y por qué sucede dicho fenómeno; permite, además, un mayor acercamiento al fenómeno desde la perspectiva de los significados que los propios actores dan al proceso en que están inmersos. Las investigaciones en salud no estuvieron ajenas al debate controversial que provocó la introducción del método cualitativo en la investigación científica pero partiendo de la base de que el conjunto de datos cuantitativos y cualitativos no se oponen ni se yuxtaponen o excluyen, la integración de ambos métodos, lejos de dificultarla puede potenciarla. La integración y convergencia de ambos métodos puede contribuir a una mejor comprensión del fenómeno en estudio. La aplicación de las ciencias sociales al estudio y comprensión de los procesos de salud/enfermedad facilitan el tránsito del enfoque biomédico al enfoque biosocial y obliga al uso de la metodología cualitativa de investigación.
\end{abstract}

Palabras clave: Investigación cuantitativa, investigación cualitativa, investigaciones en salud, ciencias sociales, diabetes mellitus.

\begin{abstract}
The objective of this paper is to comment on the possibility of integration and convergence of the quantitative and qualitative methods in health research. It also intends to characterize both methods and to illustrate them with concrete researches in the health field. The information offered is based on the bibliographic review, the theoretical exposition and the presentation of results obtained in social
\end{abstract}


and educational research in the diabetes field. Both methods have different research aims and different ways of approaching the phenomenon to be studied, its advantages and limitations. The quantitative method is useful to measure the magnitude of a phenomenon, whereas the qualitative method serves to interpret how and why this phenomenon occurs. It also allows to get closer to the phenomenon from the perspective of the meanings that the actors give to the process in which they are immersed. The health researches were not foreign to the controversial debate caused by the introduction of the qualitative method into scientific research, but starting from the basis that the set of qualitative and quatitative data do not oppose or exclude each other, the integration of both methods may potentiate scientific research instead of making it difficult. The integration and convergence of both methods may contribute to a better understanding of the phenomenon under study. The application of social sciences to the study and understanding of the health/disease processes facilitate the transfer of the bio-medical to the bio-social approach and leads to the use of qualitative methodology for research.

Key words: Quantitative research, qualitative research, health research, social sciences, diabetes mellitus.

\section{NTRODUCCI ÓN}

De todos es conocido que si bien el interés por comprender y explicar los fenómenos relacionados con el hombre y su carácter social data de la antigüedad, la investigación social, propiamente dicha, no surge hasta el siglo XIX. ${ }^{1}$

Al surgir como forma particular del conocimiento, se nutre de los métodos hasta el momento utilizados por las ciencias naturales que tomaban sus bases filosóficas del positivismo de Comte y del método empírico, ${ }^{1}$ manteniendo su predominio con los enfoques funcionalistas y estructuralistas hasta después de la Segunda Guerra Mundial (Mendoza R. Investigación cualitativa y cuantitativa. Diferencias y limitaciones.

http://www.monografía.com). Pero ya en esta misma época, bajo la influencia de los estudios etnográficos de Boas (EE.UU.) y Malinowsly (Reino Unido), ${ }^{1}$ empiezan a surgir diferentes estudios interpretativos de grupos étnicos y culturas indígenas. En la segunda mitad del siglo, a partir de los años 60, con la influencia de los cambios económicos y sociopolíticos sufridos en la época de la posguerra, se amplía el campo de investigación social para interpretar los fenómenos de la vida cotidiana de los grupos minoristas de los conglomerados urbanos (inmigrantes, grupos marginales, grupos étnicos discriminados). Se hace énfasis en la interpretación de la subjetividad del hombre, de sus valores culturales, sus representaciones sociales, las concepciones con que afronta el contexto social de su cotidianeidad y las relaciones que produce en la estructura social.

Con este nuevo enfoque, los métodos tradicionales de investigación cuantitativa van resultando cada vez más estrechos al campo de las ciencias sociales, fenómeno que da lugar al surgimiento de un nuevo paradigma de investigación, conocido en la actualidad como el método de investigación cualitativa. 
Como método, la investigación cualitativa tiene diferentes premisas, técnicas y procesos de análisis e interpretación de la información, lo cual -en sus primeros tiempos de desarrollo- dio lugar a más de una controversia y crítica científica. En los primeros tiempos el debate se centraba en las diferencias ${ }^{2}$ y podía llegar a posiciones tan extremas como negar el carácter científico y el valor de los resultados del método cualitativo, en defensa de la objetividad y posibilidad de generalización "no cuestionables" del método cuantitativo. Se consideraban métodos incompatibles toda vez que sus bases epistemológicas y ontológicas eran excluyentes.

El continuo desarrollo del campo de las investigaciones en ciencias sociales no solo ha mostrado, el valor y el rigor científico de la metodología cualitativa, sino que ha permitido determinar a cuáles preguntas de investigación da respuestas y sobre todo ha demostrado que lo cuantitativo y lo cualitativo ni se yuxtaponen ni se excluyen sino que se complementan ${ }^{2}$ y aún más, pueden integrarse y converger para el enriquecimiento de la producción de un nuevo conocimiento. ${ }^{3}$

Centrando la atención en esta última posición, el propósito del presente trabajo es abordar la integración y convergencia de ambos métodos de investigación, haciendo énfasis en su utilidad y ejemplificando con resultados de investigaciones concretas en el campo de la salud. Para ello, se propone desarrollar los siguientes objetivos: 1. Caracterizar ambos métodos y exponer los criterios que deben primar para seleccionar uno u otro en el proceso de investigación. 2. Exponer la utilización, integración y convergencia de ambos métodos en las investigaciones en salud. 3. Ejemplificar, con resultados de investigaciones, la utilidad de la combinación de ambos métodos para lograr una mejor comprensión del fenómeno en estudio.

\section{MÉTODOS}

La información expuesta a continuación se basa en la revisión bibliográfica, la exposición teórica y la presentación de resultados obtenidos en investigaciones sociales y educacionales en el campo de la salud.

\section{CARACTERI ZACI ÓN DE LOS MÉTODOS CUALITATI VO Y CUANTITATIVO DE I NVESTI GACI ÓN Y EXPOSICIÓN DE LOS CRITERIOS QUE DEBEN PRIMAR PARA SELECCI ONAR UNO U OTRO EN EL PROCESO DE I NVESTI GACIÓN}

El método cuantitativo, experimental y deductivo tiene sus premisas en el positivismo, corriente filosófica predominante en las ciencias naturales, utiliza el lenguaje objetivo de las cifras y procesos estadísticos, con énfasis en el punto de vista del investigador. Como propósito de investigación busca describir la magnitud de los fenómenos y sus causas, intenta simplificar la realidad y establecer generalizaciones empíricas. ${ }^{4}$ La metodología cuantitativa utiliza el método hipotético deductivo. Parte del planteamiento de hipótesis que se confirman o verifican mediante la obtención de evidencia empírica, tratando de eliminar el azar, ${ }^{5}$ su diseño es sistemático y apriorístico; su muestreo, estadístico. Desde su perspectiva biopositivista da un carácter único, operacional y objetivo a su información. ${ }^{5}$ Como técnicas de recogida de información utiliza los cuestionarios cerrados, la observación, las encuestas y las entrevistas estructuradas, entre otras. Realiza un procesamiento estadístico a sus datos y los presenta en tablas de contingencia y gráficos. 
Por su parte, el método cualitativo, no experimental e inductivo se basa en las corrientes filosóficas de la fenomenología y la etnografía. Utiliza un lenguaje verbal subjetivo, con un enfoque antropológico o etnológico, donde predominan los significados que los propios participantes dan al fenómeno estudiado. Tiene como propósito entender la realidad desde la óptica de sus propios actores. Busca la comprensión e interpretación del contenido, las motivaciones y las condicionantes humanas de los fenómenos. ${ }^{6}$ La metodología cualitativa se basa en el método inductivo exploratorio, lo que implica formular preguntas que se exploran y se desarrollan en el proceso de la investigación y que pueden servir para plantear hipótesis. Busca, más que "la verdad", la comprensión detallada de las perspectivas de las personas que participan en el estudio, considera que la verdad está compuesta por múltiples construcciones de la realidad más que por una realidad única y objetiva. ${ }^{7}$ En este sentido trabaja con el universo de signos, motivaciones, aspiraciones, creencias y valores buscando una interpretación más profunda de los procesos y los fenómenos. Como técnicas de recogidas de la información utiliza la observación participante, la entrevista a profundidad, las discusiones de grupos, los grupos nominales y focales, los informantes claves, las historias de vida y otras. Realiza un informe narrativo de los fenómenos que estudia. ${ }^{8,9}$

De todo lo anterior se infiere que cada uno de los métodos tiene diferente objetivo de investigación y forma de abordar el fenómeno a estudiar, y acorde con ello, cada uno tiene sus propias ventajas y limitaciones. Mientras al método cuantitativo se le critica el enfoque reduccionista del fenómeno en estudio, la rigidez apriorística de las variables a estudiar y la participación pasiva de los informantes, al método cualitativo se le identifica como puntos débiles la subjetividad del investigador, la imposibilidad de generalización, el carácter anecdótico de la presentación de la información, ${ }^{4}$ y los modos en que transforma los datos en interpretaciones que, si no se hace adecuadamente, no pueden sustentarse científicamente. ${ }^{10}$

Mientras el método cuantitativo ha resultado muy útil cuando se quiere medir la magnitud de un fenómeno, cuando se quiere realizar estudios de casos y controles y se quiere inferir desde los resultados de una muestra la generalización de un fenómeno, el método cualitativo resulta útil cuando se quiere interpretar el por qué del fenómeno en estudio desde la perspectiva de los participantes en dicho estudio.

Entonces, el criterio que debe primar en el momento de elegir un método u otro de investigación es precisamente el objetivo de la investigación, pero sin desechar la posibilidad de la integración y convergencia de ambos métodos para lograr una apreciación más completa del fenómeno estudiado.

En el recuadro se puede observar un resumen comparativo de las principales características que identifican y diferencian ambos métodos.

Recuadro. Principales características que identifican y diferencian ambos métodos

\begin{tabular}{|l|l|l|}
\hline & Método cuantitativo & Método cualitativo \\
\hline Marco Teórico & Positivismo de Comte. & $\begin{array}{l}\text { Fenomenología y } \\
\text { Etnografía. }\end{array}$ \\
\hline Fin & $\begin{array}{l}\text { Medir la cuantía del } \\
\text { fenómeno en estudio, } \\
\text { determinar sus causas. }\end{array}$ & $\begin{array}{l}\text { Interpretar y } \\
\text { comprender el } \\
\text { fenómeno en } \\
\text { estudio. }\end{array}$ \\
\hline Preguntas de & ¿Cuál es la magnitud & ¿Cuáles son las \\
\hline
\end{tabular}




\begin{tabular}{|c|c|c|}
\hline $\begin{array}{l}\text { investigación } \\
\text { que responde }\end{array}$ & $\begin{array}{l}\text { del problema? ¿Cuáles } \\
\text { son sus causas? ¿Con } \\
\text { qué variables se asocia? }\end{array}$ & $\begin{array}{l}\text { dimensiones del } \\
\text { fenómeno? } \\
\text { ¿Qué está } \\
\text { ocurriendo } \\
\text { realmente? ¿Por qué } \\
\text { surge? } \\
\text { ¿Cómo actúa el } \\
\text { fenómeno? } \\
\text { ¿Qué significado le } \\
\text { dan los involucrados } \\
\text { en él? }\end{array}$ \\
\hline $\begin{array}{l}\text { Objeto de } \\
\text { estudio }\end{array}$ & $\begin{array}{l}\text { Asociación o relación } \\
\text { entre variables } \\
\text { cuantificables. }\end{array}$ & $\begin{array}{l}\text { Contextos } \\
\text { estructurales y } \\
\text { situacionales. }\end{array}$ \\
\hline Razonamiento & $\begin{array}{l}\text { Hipotético-deductivo, } \\
\text { operacionabilidad de los } \\
\text { datos. }\end{array}$ & $\begin{array}{l}\text { Exploratorio- } \\
\text { inductivo, análisis } \\
\text { de los datos. }\end{array}$ \\
\hline $\begin{array}{l}\text { Enfoque } \\
\text { centrado en: }\end{array}$ & $\begin{array}{l}\text { La estructura, lo } \\
\text { externo y objetivo. }\end{array}$ & $\begin{array}{l}\text { Lo humano, interior, } \\
\text { subjetivo, los } \\
\text { significados de las } \\
\text { conductas. }\end{array}$ \\
\hline Perspectiva & $\begin{array}{l}\text { Parcial, fragmentada, } \\
\text { estática. }\end{array}$ & Holística, dinámica. \\
\hline Orientación & Al resultado. & Al proceso. \\
\hline Diseño & $\begin{array}{l}\text { Preestablecido, medible, } \\
\text { comprobable, replicable. }\end{array}$ & $\begin{array}{l}\text { Flexible, busca } \\
\text { entendimiento de } \\
\text { los significados y de } \\
\text { las conductas. }\end{array}$ \\
\hline Muestreo & Estadístico. & Teórico estructural. \\
\hline Técnicas & $\begin{array}{l}\text { Centradas en } \\
\text { instrumentos validados: } \\
\text { observación directa, } \\
\text { cuestionarios, encuestas } \\
\text { y entrevistas } \\
\text { estructuradas, entre } \\
\text { otras. }\end{array}$ & $\begin{array}{l}\text { Centradas en el } \\
\text { trabajo de campo: } \\
\text { observación } \\
\text { participante, } \\
\text { entrevistas a } \\
\text { profundidad, grupos } \\
\text { focales y nominales, } \\
\text { entre otras. }\end{array}$ \\
\hline $\begin{array}{l}\text { Análisis de los } \\
\text { datos }\end{array}$ & Estadístico. & I nterpretativo. \\
\hline Lenguaje & Objetivo y matemático. & $\begin{array}{l}\text { Narrativo y } \\
\text { anecdótico. }\end{array}$ \\
\hline Inferencia & $\begin{array}{l}\text { Más allá de sus datos, } \\
\text { (generalizable). }\end{array}$ & $\begin{array}{l}\text { De sus datos, (no } \\
\text { generalizable). }\end{array}$ \\
\hline Posición ética & Neutralidad. & Compromiso. \\
\hline Limitaciones & $\begin{array}{l}\text { Enfoque reduccionista y } \\
\text { fragmentado de la } \\
\text { realidad, } \\
\text { desconocimiento de } \\
\text { procesos subyacentes, } \\
\text { simplificación de la } \\
\text { realidad. }\end{array}$ & $\begin{array}{l}\text { No generalizable, } \\
\text { subjetividad, no } \\
\text { permite cálculo para } \\
\text { análisis de } \\
\text { tendencias, lenguaje } \\
\text { informal. }\end{array}$ \\
\hline
\end{tabular}


Nota: la construcción del recuadro se basó en la revisión bibliográfica realizada para la redacción del presente artículo.

\section{UTI LI ZACI ÓN, I NTEGRACI ÓN Y CONVERGENCI A DE AMBOS MÉTODOS EN LAS I NVESTI GACI ONES EN SALUD}

Las investigaciones en salud no estuvieron ajenas al debate controversial que en los años 60 provocó la introducción del método cualitativo en la investigación científica. Con una fuerte tradición de investigaciones médicas, utilizando el método experimental de los estudios epidemiológicos y los ensayos clínicos, la introducción del método cualitativo fue, durante largo tiempo, fuertemente criticada en el campo de la salud, argumentando carencia de rigor científico, ${ }^{2}$ cuando realmente es la aplicación de las ciencias sociales al estudio de la salud y la enfermedad. ${ }^{11}$

No obstante, diferentes aspectos influyeron en la introducción del método cualitativo en las investigaciones en salud. Así se puede citar el interés de los científicos sociales en las investigaciones sanitarias y la necesidad de abordar determinados problemas de salud desde perspectivas bien diferentes a las biomédicas, ${ }^{8}$ la necesidad de avanzar en el conocimiento de las necesidades y expectativas de salud de los individuos así como los factores que intervienen en la efectividad de los programas de intervención en salud, ${ }^{12}$ en el estudio de las enfermedades no transmisibles donde se impone ir a la escucha de los sentimientos, significados, barreras percibidas de los que sufren la enfermedad; ${ }^{10,13}$ cuando es necesario re-contextualizar al sujeto y la complejidad de la realidad y donde el ensayo clínico y el metaanálisis no resultan suficientes. ${ }^{12}$ Los estudios de servicios en la atención primaria de salud ${ }^{14}$ son, entre otros, ejemplos de la necesidad de incorporar la metodología cualitativa como complemento necesario de los tradicionales métodos estadísticos-epidemiológicos en salud ${ }^{15,16}$ y apoyan el auge que ha tomado lo cualitativo en el campo de la salud pública y de las investigaciones en servicios de salud. ${ }^{12}$

En sus inicios, esta introducción no estaba libre de mitos. Se pensaba que la investigación cualitativa solo servía como paso previo a la construcción de un cuestionario, como exclusiva del campo de la promoción en salud. ${ }^{11}$ Ya en la actualidad, la metodología cualitativa en las ciencias de la salud se utiliza para explorar fenómenos de los que se sabe poco, para describir dimensiones y variaciones de los fenómenos en estudio, para analizar las características generales de un fenómeno mal comprendido, para comprender cómo o porqué ocurre el fenómeno, ${ }^{8}$ para conocer las preferencias de los usuarios de los servicios de salud, entre otros. ${ }^{17}$ Así, se encuentra un considerable número de artículos científicos en diferentes temas de salud basados en la investigación cualitativa. ${ }^{18-22}$ (Inés RM. Paradigmas de investigación en salud 2002. monografía.com).

Sin embargo, hay todavía otra alternativa. Si se parte de la base de que el conjunto de datos cuantitativos y cualitativos no se oponen, sino que se complementan, ${ }^{22}$ si se tienden puentes entre el método cuantitativo y cualitativo, ${ }^{2}$ si cuando el objetivo de investigación así lo requiere, se hace coincidir en un mismo estudio la integración de ambos métodos, el uso conjunto de ambas metodologías lejos de dificultar la investigación puede potenciarla ${ }^{23}$ con el empleo convergente y racional de $\operatorname{ambos}^{17}$ y se tendrá una buena herramienta para incursionar en los temas de la salud publica. 


\section{EJ EMPLI FI CACIÓN DE LA UTI LI DAD DE LA COMBI NACIÓN DE AMBOS MÉTODOS PARA LOGRAR UNA MEJ OR COMPRENSIÓN DEL FENÓMENO EN ESTUDIO}

Dentro de la amplia gama del proceso salud/enfermedad, las enfermedades no transmisibles ocupan, en estos tiempos, un lugar relevante por el constante ascenso de su prevalencia e incidencia en la población adulta, así como por el lugar que ocupan dentro de las principales causas de muerte. ${ }^{24,25}$ (García R, Suárez R. 2001. Modelo teórico para la educación terapéutica desde la experiencia en personas con diabetes. Paho.org/spanish/AD/DPC/NC/dia-cuba.pdf). Por otra parte, la cronicidad y las limitaciones que estas enfermedades imponen, hacen que las enfermedades crónicas no transmisibles sean un ejemplo de la necesidad de este abordaje integral, ${ }^{26}$ (Assal J Ph. From biomedicine to patient needs: global, regulatory, cybernetic challenge. Some unanswered questions and possible solutions. X Masletter-Claude Bernard. Doc. Dec 2000), donde se recupere la voz de quienes padecen: la persona con la enfermedad, el familiar, los amigos, los compañeros de trabajo ${ }^{13}$ y se desarrolle un proceso de educación continuada para que las personas aprendan a vivir con calidad de vida. Dentro de ellas, la diabetes mellitus (DM) es un buen ejemplo porque requiere de un tratamiento para toda la vida y de un seguimiento periódico, principal vía para evitar la aparición de complicaciones agudas y crónicas de esta enfermedad, propias del mal control metabólico. ${ }^{27}$ Las exigencias de esta enfermedad pueden interferir o ser interferidas por la actividad diaria del paciente; sin embargo no son frecuentes los estudios que aborden los problemas sociales de las personas con diabetes.

En la experiencia de los autores, con la educación y el trabajo social dirigidos a personas con diabetes, la combinación de técnicas cuantitativas y cualitativas en un mismo proyecto de investigación ha sido muy útil para determinar la magnitud del problema (cuantitativo) y, a su vez, comprender por qué se comporta de una u otra manera (cualitativo).

La evaluación, durante más de 25 años, del programa cubano de educación en diabetes se inició en los años 80 con investigaciones de corte cuantitativo. ${ }^{28,29}$ Ya en los años 90 se incorporó el método cualitativo integrado con el cuantitativo y como ejemplo de ello se puede citar la evaluación de la influencia de un proceso de educación interactiva en los conocimientos, destrezas y adhesión al tratamiento así como su repercusión en el control metabólico y en algunos sentimientos y conductas relacionados con la DM en diabéticos tipo $1 .^{30,31}$ Tanto en el corte de 5 años como en el de 10 años se pudo constatar que el cuestionario de sentimientos y conductas (técnica cuantitativa) brindó una puntuación continua de cómo iban avanzando las respuestas emocionales ante las exigencias terapéuticas, mientras las discusiones de grupo (técnica cualitativa) permitió profundizar en el significado que los participantes daban a sus respuestas conductuales y a su sentir sobre el afrontamiento de la diabetes.

En estudio similar, con seguimiento de 5 años en personas con diabetes mayores de 60 años con diseño experimental sucesivo, ${ }^{32}$ se integraron técnicas cualitativas de evaluación y se pudo alcanzar una mejor comprensión de los sentimientos, criterios y creencias de salud con respecto a la enfermedad, así como identificar las barreras percibidas al afrontar el autocuidado diario.

Para el proceso de generalización del programa de educación en diabetes a los servicios de la atención primaria de salud se realizó una investigación nacional ${ }^{33}$ donde también se hicieron converger ambos métodos y las técnicas cualitativas que sirvieron de base para el diagnóstico participativo facilitaron la identificación y el 
acercamiento entre proveedores de salud y personas con diabetes. Al triangular la información se encontró que mientras el proveedor de salud no identificaba la función emisora del paciente, estos sí consideraban la posibilidad de ayudar a otros desde su propia experiencia y reconocían la relación de pares como un espacio de aprendizaje, aspectos que no surgieron en los instrumentos de corte cuantitativo.

Más recientemente, en el año 2008, se terminó otra investigación de carácter descriptivo exploratorio donde se integraron ambos métodos con el fin de lograr un acercamiento a la problemática laboral de un grupo de personas atendidas en el Centro de Atención al Diabético del Instituto Nacional de Endocrinología. ${ }^{34}$ En dicho estudio, al triangular los resultados de encuestas y discusiones de grupos se obtuvo una mayor riqueza y mejor interpretación de la información recogida; así mientras menos de la tercera parte $(26,6 \%)$ de los entrevistados declaró que la DM afectaba el desempeño laboral o el cumplimiento del trabajo obstaculizaba el buen control de la DM, en las discusiones grupales, de una manera u otra, todos reconocían que había una interdependencia entre estos fenómenos. Por otra parte, tal como se encontró en $1984,{ }^{35}$ la DM no predominó como causa de jubilación en el dictamen de peritaje, no obstante, el presente estudio reveló que jubilados que se acogieron a dicho proceso, haciendo uso de sus derechos de edad y tiempo laborado, durante la discusión expresaban que si hubieran tenido otras condiciones o una mayor comprensión en su centro laboral, se hubieran mantenido activos y que tomaron la decisión por ver afectada su salud.

Todos estos hechos en investigaciones concretas en el campo de la salud son ejemplo de que la práctica investigativa va relegando las posiciones que consideran que ambos métodos se excluyen y abre paso a una posición científica más flexible, siempre colocando en el centro de la discusión el objetivo de investigación, aspecto determinante para la selección de uno u otro método o para la alternativa de integrarlos en un mismo proyecto de investigación.

También resulta inaceptable en la actualidad la afirmación de que la metodología cualitativa carece de objetividad y rigor científico. La falta de objetividad y rigor científico puede estar presente en los informes de cualquiera de ambos métodos por mal procedimiento investigativo o por mal procesamiento de la información adquirida y cada uno de estos métodos aporta, desde sus raíces teóricas y en sus diferentes estilos de procesamiento y presentación de los datos, una herramienta útil para la búsqueda del conocimiento, para la medición, comprensión o interpretación de los fenómenos en estudio.

\section{CONSI DERACI ONES FI NALES}

La existencia de dos paradigmas de investigación diferentes, no implica una yuxtaposición o exclusión entre ellos. No hay uno mejor que el otro. La clave está en que el investigador sepa tomar partido de acuerdo a sus preguntas y objetivo de investigación.

El método cualitativo enriquece el campo de la investigación social, pues permite un mayor acercamiento al fenómeno en estudio, desde la perspectiva de los significados que los propios actores dan al proceso en que están inmersos. Dada la complejidad del estudio del fenómeno social, la integración y convergencia de ambos métodos puede contribuir a una visión más holística y una mejor comprensión del fenómeno en estudio.

En la medida en que las investigaciones de salud, se centren menos en el proceso salud-enfermedad para ubicarse en el hombre que desarrolla ese proceso y su 
calidad de vida, en la medida en que se desarrolla la salud pública y se hacen más necesarias las evaluaciones de diagnóstico y de desarrollo de programas de intervención así como las evaluaciones de sistemas y servicios de salud; se hace más necesaria la incorporación del método cualitativo como antecedente exploratorio, descriptivo o explicativo que complemente al método cuantitativo y permita la mejor comprensión e interpretación del fenómeno en estudio. La aplicación de las ciencias sociales al estudio y comprensión de los procesos de salud enfermedad facilitan el tránsito del enfoque biomédico al enfoque biosocial, lo que obliga al uso de la metodología cualitativa de investigación en algún momento del desempeño profesional.

\section{REFERENCI AS BI BLI OGRÁFICAS}

1. Rojo N. La investigación cualitativa. Aplicaciones en salud. La Habana: Escuela Nacional de Salud Pública; 2003. [Dossier de la Maestría en Administración de Salud].

2. Romo M, Castillo D. Metodología de las Ciencias Sociales aplicadas al estudio de la nutrición. Rev Chilena Nut. 2002;29.

3. Martínez M. La investigación cualitativa (Síntesis conceptual). Revista IIPSI Facultad de Psicología UNMSM. 2006; 9: 123.

4. Bacallao J, Alerm A, Artiles L. La integración de los métodos cuantitativos y cualitativos en la investigación bio-social. La Habana: Escuela Nacional de Salud Pública;2002. [Texto complementario de metodología de la investigación educacional. Material de la Maestría de Educación Médica].

5. Cabrera L, Reichart M. El debate investigación cualitativa frente a investigación cuantitativa. Enfermería clínica. 1996;6:212.

6. Ulin PR, Robinson ET, Tolley EE. Investigación aplicada en Salud Pública. Método cualitativo. Washington: OPS; 2006. [Publicación científica No. 614.Cap. 2. p.16].

7. Salamanca $A B$. La investigación cualitativa en las ciencias de la salud. Nure Investigación. 2002;(24).

8. Pita S, Pertezas S. Investigación cuantitativa y cualitativa. CAD Atención Primaria. 2002; 9: 76.

9. de Souza Ma.C. Ciencia, técnica y arte: el desafío de la investigación social. En: Investigación social. Teorías, métodos y creatividad. Buenos Aires: Editorial Buenos Aires; 2004.

10. Amezcua M, Gálvez A. Los modos de análisis en investigación cualitativa en salud: perspectiva crítica y reflexiones en voz alta. Rev Española Salud Pública. 2002; 76: 423.

11. Frenk J. La nueva Salud Pública. En: La crisis de la Salud Pública. Reflexiones para el debate. Washington: OPS; 1992.

12. Calderón C. Criterios de calidad en la investigación cualitativa en salud (ICS): Apuntes para un debate necesario. Rev Española Salud Pública. 2002; 76:5. 
13. Mercado FJ . Investigación cualitativa en América Latina: Perspectiva crítica en Salud. Inter J Qualitative Methods. 2002; 1. [Article 4. Retrieved Date from http://www. ualberta.ca/ ijam/].

14. Bayarre Vea H, Hosford Saing R, editores. Métodos y técnicas aplicadas a la investigación en Atención Primaria de Salud [sitio en Internet]. [citado 30 Oct 2007]. Disponible en:

http://www.sld.cu/galerias/pdf/sitios/dne/bayarre_y_hosford.pdf

15. Conde F, Pérez A. La investigación cualitativa en Salud Pública. Rev Española Salud Pública. 1995; 69: 145.

16. Boum F. Investigaciones en Salud Pública. El debate sobre la metodología cuantitativa y cualitativa. Rev Española Salud Pública. 1997;5:175.

17. Carbonell Ma.A, Aranaz JM, Mira JJ, Pérez V, García A. La técnica cualitativa en la mejora de la satisfacción en urgencias. Emergencias. 2004; 6: 184.

18. Gil M, Estrada C, Pérez MI, Aguirre R. La investigación cualitativa y la promoción de salud en la comunidad de Madrid. Rev Española Salud Pública. 2002; 76: 451.

19. Grypdonck MHF. Qualitative Health Research in the Era of Evidence-Based Practice. Qualitative Health Res. 2006; 16:1371.

20. Ramos BN. Enfoque conceptual y de procedimientos para el diagnóstico y análisis de la situación de salud. Rev Cubana Salud Pública. 2006; 32(2).

21. Louro BI. Modelo de salud del grupo familiar Rev Cubana Salud Pública. $2005 ; 31(1)$.

22. Álvarez L, Cabrera A (compiladoras). La investigación en Salud Sexual y Reproductiva. Propuestas metodológicas y experiencias. La Habana: Publicaciones Azucareras; 2004.

23. Cook $\mathrm{CH}$. Métodos cualitativos y cuantitativos en investigación evaluativa. Madrid: Morata; 1986.

24. Département de l'action sociale et de la santé. Planification qualitative du systéme de santé genovois. Rapport 1: les domaines d'actions prioritaires et la réforme du systéme de santé. Introduction. Les Cahiers de la Santé. 1998(91): 9.

25. Assal J. Revisting the approach to treatment of long term illness: from the acute to the chronic state. A need for educational and managerial skills for long term follow-up. Patient Educ Counsell. 1999; 37: 99.

26. From metabolic crisis to long term diabetes continuous. A plea for more efficient therapy. In: Davidson JK, editor. Clinical Diab Mell. A problem oriented approach. 3rd ed. New York: Thième;2000.p.799.

27. Macías M, Pérez L. La Diabetes Mellitus y sus complicaciones vasculares: un problema social de salud. Rev Cubana Angiología. Cir Vas. 2003; 1:68. 
28. García R, Suárez R. Confiabilidad y validez de un cuestionario de conocimientos en Diabetes. Rev Cubana Endocr. 1991;1:16.

29. . La educación del paciente diabético. La Habana: Editorial Ciencias Médicas; $19 \overline{9} \overline{2}$. [Premio Latinoamericano al mejor trabajo de atención y educación a diabéticos. 2da. reimpresión].

30. García R, Suárez R, Gómez J, Romero MI, Portilla L. Educación interactiva vs. educación convencional en diabéticos insulina-dependientes. Rev Avances Diabetología. 1994; 9:45.

31. García R, Suárez R. Continuous care and education for type 1 diabetic patients. One to one consultations or group sessions. Diabetología. 1999; 42: A 256,967.

32. Diabetes Education in the elderly: a 5 -year follow up of an interactive approach. Patient Educ Counsell. 1996;29:87.

33. Diagnóstico de la situación de educación en diabetes en la Atención Primaria. Rev Cubana Endocr. 1997; 8:65.

34. García R, García M, Suárez R, Domínguez E. Problemática laboral en personas con diabetes mellitus atendidas en el centro diurno del centro de atención al diabético. Rev Cubana Endocr. En prensa 2009.

35. García R, Suárez R, Aguíar M. Procesos de peritajes en pacientes diabéticos. Rev Cubana Salud Pública. 1992; 18: 95.

Recibido: 6 de febrero de 2009.

Aprobado: 23 de junio de 2009.

Rosario García González. Instituto Nacional de Endocrinología. Calle 17 esquina a D. El Vedado municipio Plaza. Ciudad de La Habana, Cuba.

* Presentado en el Simposio "Arnaldo Tejeiro Fernández", Investigación Cualitativa en Salud Pública, 23 de junio de 2009. 